\title{
GROUPS WITH AN ABELIAN SYLOW SUBGROUP
}

\author{
JOHN W. BALLARD ${ }^{1}$
}

\begin{abstract}
The purpose of this note is to obtain a modular refinement of Brauer's induction theorem for groups with an abelian Sylow subgroup.
\end{abstract}

1. Introduction. Let $\chi$ be a complex irreducible character of a finite group $G$. Brauer's induction theorem shows that $\chi$ may be expressed as an integer linear combination of characters induced from elementary subgroups of $G$. The object of this note is to show that if a Sylow $p$-subgroup of $G$ is abelian, then $\chi$ may be expressed as an integer linear combination of characters induced from elementary subgroups whose $p$-part is contained in the $p$-defect group of $\chi$.

2. Notation. For $H$ a finite group let $\operatorname{ch}(H)$, the ring of generalized characters, be the set of integer linear combinations of the complex irreducible characters of $H$. If $\chi$ and $\psi$ are elements of $\operatorname{ch}(H),(\chi, \psi)_{H}$ denotes their usual inner product.

A group $E$ is elementary if it is the direct product of a $q$-group and a cyclic $q^{\prime}$-group, for some prime $q$. Note that for any elementary group $E$, we may write $E=A \times B$ where $A$ is a $p$-group and $B$ is a $p^{\prime}$-group. The subgroup $A$ is referred to as the $p$-part of $E$.

For $g \in G$, let $g_{p}$ denote the $p$-part of $g$. The $p$-defect group of an irreducible character is a defect group of the $p$-block which contains the character.

3. General case. Let $P$ be a Sylow $p$-subgroup of $G$. In this section we assume that $D$ is a subgroup of $P$ which satisfies the following two conditions.

(i) For any $g \in G, D^{g} \cap P \subseteq D$.

(ii) For any subgroup $A \subseteq P$, the restriction homomorphism of $\operatorname{ch}(A)$ into $\operatorname{ch}(A \cap D)$ is surjective.

If $E=A \times B$ is an elementary subgroup of $G$, let $A^{\prime}=A \cap D$ and $E^{\prime}=A^{\prime} \times B$.

Proposition. Let $\chi$ be an element of $\operatorname{ch}(G)$ such that $\chi(g)=0$ if $g_{p}$ is not conjugate to an element of $D$. Then $\chi$ may be expressed as an integer linear combination of characters induced from elementary subgroups whose p-part is contained in $D$.

Received by the editors April 22, 1977.

AMS (MOS) subject classifications (1970). Primary 20C15; Secondary 20 C20.

'Research partially supported by a National Science Foundation grant. 
Step 1. Let $E=A \times B$ be an elementary subgroup with $A \subseteq P$. Then $\mid E$ : $\left.E^{\prime}\right|^{-1} \chi_{E^{\prime}}$ is a generalized character of $E^{\prime}$.

Proof. It suffices to show that $\left|E: E^{\prime}\right|^{-1}(\chi, \theta)_{E^{\prime}}$ is an integer for any irreducible character $\theta$ of $E^{\prime}$.

Now $A \subseteq P$ so by hypothesis (i), $a \in A$ is $G$-conjugate to an element of $D$ if and only if $a \in A^{\prime}$. Since $\chi$ vanishes on any element whose $p$-part is not conjugate to an element of $D$, it follows that $\chi_{E}$ vanishes on any element not in $E^{\prime}$. So for $\psi \in \operatorname{ch}(E)$

$$
(\chi, \psi)_{E}=\left|E: E^{\prime}\right|^{-1}(\chi, \psi)_{E^{\prime \prime}}
$$

Any irreducible character of $A^{\prime} \times B$ is of the form $\alpha \times \beta$, where $\alpha$ and $\beta$ are irreducible characters of $A^{\prime}$ and $B$ respectively. By hypothesis (ii), $\alpha$ is the restriction to $A^{\prime}$ of some generalized character of $A$. It follows that the restriction homomorphism of $\operatorname{ch}(E)$ into $\operatorname{ch}\left(E^{\prime}\right)$ is surjective. Choosing $\psi \in$ $\operatorname{ch}(E)$ such that $\psi_{E^{\prime}}=\theta$ in the preceding equation establishes the result.

Step 2. Let $E=A \times B$ be an elementary subgroup with $A \subseteq P$. If $\psi \in$ $\operatorname{ch}(E)$, then

$$
\left(\psi \chi_{E}\right)^{G}=\left|E: E^{\prime}\right|^{-1}\left((\psi \chi)_{E^{\prime}}\right)^{G} .
$$

Proof. For $g \in G$ we have

$$
\left(\psi \chi_{E}\right)^{G}(g)=|E|^{-1} \sum_{x \in G} \psi^{o}\left(g^{x}\right) \chi\left(g^{x}\right)
$$

where $\psi^{\circ}$ is defined by $\psi^{\circ}(y)=\psi(y)$ if $y \in E$ and 0 otherwise. Now if $y \in E$, then $y_{p} \in A$ and again by hypothesis (i), $\chi(y)=0$ if $y_{p} \notin A^{\prime}$. So

$$
\chi \psi^{o}=\chi\left(\psi_{E^{\prime}}\right)^{o}
$$

and the result then follows from the definition of the induced character.

Step 3. We now complete the proof of the proposition. By Brauer's induction theorem [2, Theorem 16.2] we may write

$$
1=\sum a_{i} \psi_{i}^{G}
$$

where the $a_{i}$ are integers and the $\psi_{i}$ are linear characters of elementary subgroups $E_{i}$. Write $E_{i}=A_{i} \times B_{i}$. Replacing $E_{i}$ by a conjugate subgroup if necessary, we may assume that $A_{i} \subseteq P$.

From (*) we have

$$
\chi=\sum a_{i}\left(\psi_{i} \chi_{E_{i}}\right)^{G}
$$

and by Step 2

$$
\left(\psi_{i} \chi_{E_{i}}\right)^{G}=\theta_{i}^{G} \text {, where } \theta_{i}=\left|E_{i}: E_{i}^{\prime}\right|^{-1}\left(\psi_{i} \chi\right)_{E_{i}^{\prime}} .
$$

Now by Step $1, \theta_{i}$ is a generalized character of $E_{i}^{\prime}$, which proves the proposition.

REMARK. In equation (*) it is easy to see that there is some elementary subgroup $E_{i}$ with $A_{i}=P$. So the corresponding $\theta_{i}$ which appears in the 
expression for $\chi$ is a generalized character of an elementary subgroup whose $p$-part equals $D$.

4. Applications. We first apply the proposition with $D=1$ to obtain the following result of Brauer [1, Theorem 5].

CoRollary 1. Suppose that $\chi \in \operatorname{ch}(G)$ is such that $\chi(g)=0$ if $g_{p} \neq 1$. Then $\chi$ is an integer linear combination of characters induced from elementary subgroups whose order is prime to $p$.

Note that if $\chi$ is a principal indecomposable character for the prime $p$, then $\chi$ satisfies the hypothesis of the corollary.

For $D$ a $p$-subgroup of $G$, let $\operatorname{ch}_{D}(G)$ denote the elements of $\operatorname{ch}(G)$ which are integer linear combinations of characters of $p$-adic indecomposable representations with vertex contained in $D$. Note that if $\chi \in \operatorname{ch}_{D}(G)$, then $\chi$ vanishes on any element of $G$ whose $p$-part is not conjugate to an element of $D$ [2, Lemma 59.5]. Moreover, if $\chi$ is an irreducible character of $G$ with $p$-defect group $D$, then a vertex for $\chi$ is contained in $D$ and hence $\chi \in$ $\operatorname{ch}_{D}(G)$. So the result stated in the introduction is a consequence of the following corollary.

Corollary 2. Suppose that a Sylow p-subgroup of $G$ is abelian. Let $D$ be a $p$-subgroup of $G$. Then every element of $\operatorname{ch}_{D}(G)$ is an integer linear combination of characters induced from elementary subgroups whose p-part is contained in $D$.

Proof. We use induction on $|D|$. If $|D|=1$, the result follows from Corollary 1.

In general, if $\chi \in \operatorname{ch}_{D}(G)$, then the Green correspondence [3, Theorem 2] applied to $G, N=N_{G}(D)$ and $D$ shows that

$$
\chi-\psi^{G} \in \sum_{g \notin N} \operatorname{ch}_{D \cap D^{8}}(G)
$$

for some $\psi \in \operatorname{ch}_{D}(N)$. For $g \notin N$, the induction hypothesis yields the result for $\operatorname{ch}_{D \cap D^{8}}(G)$. Since a Sylow $p$-subgroup of $N$ is abelian, $D$ satisfies the two conditions of $\$ 3$ with respect to the group $N$. Now applying the proposition to the group $N$ and the character $\psi$ yields the result for $\psi$ and hence completes the proof of the corollary.

It would be interesting to know to what extent the analog of Corollary 2 holds for an arbitrary finite group.

The author would like to thank the referee for his helpful suggestions.

\section{REFERENCES}

1. R. Brauer, Applications of induced characters, Amer. J. Math. 69 (1947), 709-716. MR 9, 268.

2. I. Dornhoff, Group representation theory, Parts A and B, Marcel Dekker, New York, 1971. MR 50 \# 458.

3. J. A. Green, A transfer theorem for modular representations, J. Algebra 1 (1964), 73-84. MR 29 \# 147.

Department of Mathematics, University of Washington, Seattle, Washington 98195 